\title{
Inter-commodity Arbitrage Based on Stochastic Process Model
}

\author{
Quan Gu*, Xinghui Lei \\ School of Economics and Management, Tongji University, Shanghai, 200092, China \\ *11guquan@tongji.edu.cn
}

Keywords: Ornstein-uhlenbeck Process, Statistical Arbitrage, Futures Spread Trading.

\begin{abstract}
This paper mainly discusses statistical arbitrage model in the application of agriculture futures inter-commodity arbitrage. Based on the substitution relationship, three arbitrage strategies have been designed. Different from the traditional threshold setting method, this paper puts forward the optimization idea of transaction threshold setting. The stochastic spread model based on Ornstein-uhlenbeck process is introduced into the threshold setting. The empirical results show that the feasibility of this improvement.
\end{abstract}

\section{Introduction}

In recent years, the statistical arbitrage model method is applied to the research of commodity arbitrage. Different from stock market, there are short selling restrictions on margin trading, the trading mechanism of futures market makes statistical arbitrage more applicable. Futures arbitrage dependents on the accurate portrayal of statistical arbitrage model. The key factor for the application of statistical arbitrage model in inter-commodityarbitrage is the transaction threshold setting. Most of the existing statistical arbitrage models are based on cointegration regression[1,2]. Although certain achievements have been made inimprovingmethods [3,4].But the basic premise is that residuals obey normal distribution, which often exists fat-tailed distribution. Inaccurate characterizationofresidual mean reversion will make the threshold estimation biaswhich affect the effect of arbitrage. In order to solve this problem, this paper proposes to use a new method based on Ornstein-Uhlenbeck (O-U) process to describe the volatility of the spread, and then to set the transaction threshold. Previous research has pointed out that the advantage of the continuous time model with the stochastic regression of spread could more accurately describe the characteristics of the mean return[5].Later studiesdeduced the optimal solution of the transaction threshold based on $\mathrm{O}-\mathrm{U}$ process[6].But the application of the method in futures arbitrage is still to be verified. This paper will take empirical tests based on O-U process setting method to improve threshold setting.

\section{Method}

The O-U process, as the diffusion process of physics, has the characteristic of the odd markov process. The following $\mathrm{O}-\mathrm{U}$ process is based on cointegration regression residual decentralization:

$$
\begin{array}{r}
e_{t}=\operatorname{spread}_{t}-\operatorname{Mean}\left(\operatorname{spread}_{t}\right)=\mathrm{y}_{\mathrm{t}}-\beta_{2} \mathrm{x}_{\mathrm{t}}-\beta_{1}(1) \\
\mathrm{d} e_{t}=-\alpha e_{t}+\eta d W_{t}(2)
\end{array}
$$

Where $\beta_{1}, \beta_{2}, e_{t}$ are cointegration coefficients and residuals, spread $_{t}$ is the spread sequence of cointegration regression, Mean $\left(\operatorname{spread}_{t}\right)$ is the mean of the spread sequence, and $\alpha>0, \eta>0, W_{t}$ is the wiener process.According to ITO's theorem, parameter changes can be obtained:

$$
e_{t+\Delta t}=\exp (-\alpha \cdot \Delta t) e_{t}+\eta \int_{t}^{t+\Delta t} \exp [-\alpha(t+\Delta t-s)] d W(s)(3)
$$

Let $\mathrm{b}=\exp (-\alpha \cdot \Delta t), \varepsilon=\eta \int_{t}^{t+\Delta t} \exp [-\alpha(t+\Delta t-s)] d W(s)$, the expressions can be transformed into: $e_{t+\Delta t}=b e_{t}+\varepsilon . e_{t}$ as a self-regression process: $e_{n+1}=b e_{n}+\xi_{n}$ The model 
parameters can be estimated by undetermined coefficient method and obtained:Variance $(\xi)=$ $\operatorname{Variance}(\varepsilon)=\eta^{2} \frac{1-\exp (-2 \alpha \cdot \Delta t)}{1-2 \alpha}$, then:

$$
\alpha=\frac{-\ln (b)}{\Delta t}, \eta=\sqrt{\frac{\operatorname{Variance}(\varepsilon) 2 \alpha}{1-b^{2}}}(4)
$$

According to Bertram's derivation [2], we can construct the optimal threshold solution and design the arbitrage strategy.

$$
\mathrm{a}^{*}=-\frac{c}{4}-\frac{c^{2} \alpha}{4\left(c^{3} \alpha^{3}+24 c \alpha^{2} \eta^{2}-4 \sqrt{3 c^{4} \alpha^{5} \eta^{2}+36 c^{2} \alpha^{4} \eta^{4}}\right)^{1 / 3}}-\frac{\left(c^{3} \alpha^{3}+24 c \alpha^{2} \eta^{2}-4 \sqrt{3 c^{4} \alpha^{5} \eta^{2}+36 c^{2} \alpha^{4} \eta^{4}}\right)^{1 / 3}}{4 \alpha}(5)
$$

Although we have given the corresponding transaction thresholds in the previous derivation, how to set the transaction stop loss threshold is still a problem. This article refers to previous research [7,8], according to the principle of VaR analysis, 1.5 times the standard deviation as a trade stop loss threshold, used to prevent trading risks. In addition, in order to compare the effectiveness of the new method, we used the empirical results of the traditional thresholding method as a reference group.

\section{Results}

The empirical research in this paper is on agricultural product futures. Soybean, soybean meal and soybean oil futures are listed on the Dalian Commodity Exchange. Rapeseed, rapeseed meal and vegetable oil futures are listed on the Zhengzhou Commodity Exchange. The transaction data is the daily closing price of each product from January 4, 2013 to December 30, 2016. It excludes some incomplete dates and ensures the availability of synchronized data. There are a total of 971 samples. We use the training period from January 4, 2013 to December 31, 2014 as a sample of 483 samples. We use the 483 samples from January 5, 2015 to December 30, 2016 as an out-of-sample test period. Since the futures contracts have durations, we extend the month before the conversion of the main contract to form a continuous time series of prices. Our data comes from the wind database. According to the method proposed in the previoussection, we test the arbitrage portfolio by co-integration, and describe the volatility of the spread according to the residual of the cointegration regression. The statistical characteristics of the spread are shown in Table 1.

The threshold of traditional non-parametric method can be calculated directly by the standard deviation from Table1.The threshold setting of the O-U method can be calculated by the formula. The specific calculation result is shown in Table 2. The a and $\mathrm{m}$ are the optimal transaction thresholds.

From the empirical results of the simulation transactions disclosed in Table 3, the use of the O-U method to improve the threshold setting has played a positive feedback effect on transactions. From the perspective of the average annual return, the effect of threshold setting improvement is not reflected. In theoil meal arbitrage strategy and the plant oil arbitrage strategy, the traditional non-parametric method is better. However, in the two risk-adjusted measurement of Sharpe ratio and Calmar ratio, the use of O-U method to improve the effect is more obvious. This is roughly similar to the previous study [9,10]. This is mainly because the threshold range set by the O-U method is even narrower, which increases the trading opportunities and decrease the transaction risks. Without considering transaction costs, it does improve the risk-adjusted returns of trading strategies. This point can also be confirmed from the comparison of the maximum withdrawal. In the same way, the $\mathrm{O}-\mathrm{U}$ method increases the number of transactions, reduces the average trading days, thus improves the efficiency of the trading strategy, although this improvement is not particularly noticeable.

Figure1 shows the cumulative returns of arbitrage strategies, the cumulative returns of plant oil arbitrage and oil meal arbitrage which both usenon-parametric methodare the highest and lowest respectively. The cumulative returns of the other four strategies are relatively close. From the 
comparison of different threshold setting models, the oil seed arbitrage strategy and oil meal arbitrage strategy that use O-U method are better. Judging from the comparison of different arbitrage strategies, the arbitrage strategy's cumulative return has a positive correlation with the futures price correlation.

Table 1 The statistical characteristics of the arbitrage portfolios spreads

\begin{tabular}{llll}
\hline Portfolio & ARS & YOI & MRM \\
Mean & 0.0000 & 0.0000 & 0.0000 \\
Median & 58.64146 & 42.71498 & 1.730635 \\
Maximum & 398.7295 & 609.8112 & 93.58451 \\
Minimum & 767.7695 & 985.7485 & 350.8877 \\
Standard deviation & 241.7843 & 301.5164 & 34.98835 \\
Skewness & 1.394447 & 0.895444 & 2.725303 \\
Kurtosis & 4.587392 & 3.786643 & 26.77141 \\
\hline
\end{tabular}

Note: ARS, YOI, MRM stand for oilseeds, plant oil and oil meal arbitrage portfolio respectively.

Table 2 The Value of the O-Umethod parametersfor the arbitrage portfolios

\begin{tabular}{lllllll}
\hline Portfolio & \multicolumn{1}{c}{$b$} & \multicolumn{1}{c}{ Variance $(\varepsilon)$} & \multicolumn{1}{c}{$\alpha$} & \multicolumn{1}{c}{$\eta$} & \multicolumn{1}{c}{$\mathrm{a}$} & \multicolumn{1}{c}{$\mathrm{m}$} \\
\hline ARS & 0.843 & 135.7652 & 9.4211 & 188.6245 & 35.0609 & 35.0609 \\
YOI & 1.4235 & 189.2574 & 15.0217 & 251.0189 & 77.0124 & 77.0124 \\
MRM & 1.2158 & 32.1568 & 3.2548 & 41.2045 & 5.2556 & 5.2556 \\
\hline
\end{tabular}

Table 3 Performance of the arbitrage portfolios

\begin{tabular}{lllllll}
\hline Portfolio & \multicolumn{2}{c}{ ARS } & \multicolumn{2}{c}{ YOI } & \multicolumn{2}{c}{ MRM } \\
\cline { 2 - 7 } & OUM & NPM & OUM & NPM & OUM & NPM \\
\hline Annual return & 0.254 & 0.202 & 0.205 & 0.290 & 0.220 & 0.249 \\
Sharpe ratio & 0.325 & 0.245 & 0.675 & 0.459 & 0.628 & 0.158 \\
Calmar ratio & 0.421 & 0.557 & 0.327 & 0.731 & 0.415 & 0.234 \\
Maximum withdrawal & $29.25 \%$ & $48.12 \%$ & $17.13 \%$ & $33.15 \%$ & $52.12 \%$ & $71.28 \%$ \\
Transactions & 32 & 24 & 27 & 25 & 21 & 19 \\
Average trading days & 18 & 16 & 23 & 31 & 32 & 37 \\
\hline
\end{tabular}

Note: OUM is an abbreviation of O-U method, and NPM is an abbreviation of non-parametric method.

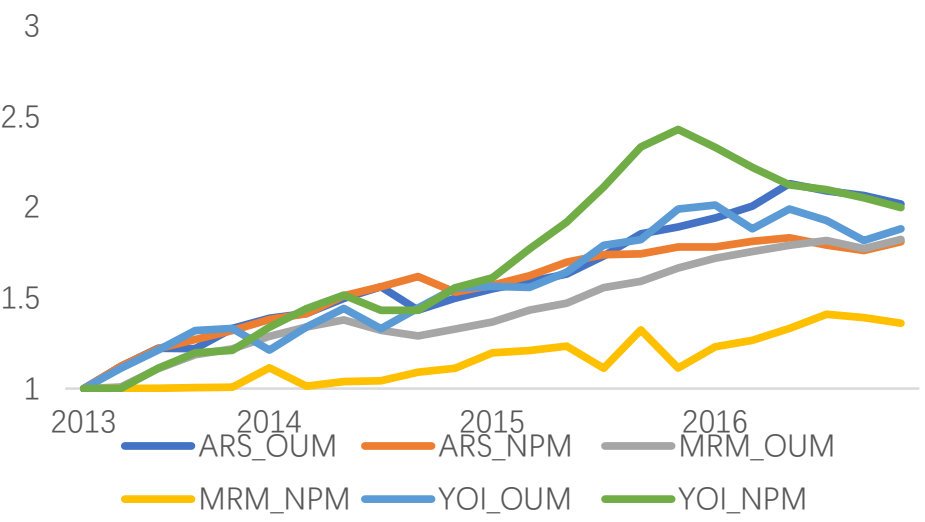

Figure1 The cumulative returns of arbitrage strategies

\section{Conclusion}

This paper discusses statistical arbitrage using stochastic process model to improve the trading threshold setting, and validates the feasibility of this model's improvement by applying three inter-commodity arbitrage strategies. The empirical results show that, consistent with most studies [11], this threshold setting improvement is also effective in inter-commodity arbitrage applications, but the significance is not sound. The main reason for this phenomenon may be that the sample space is not large enough. Future studies may adopt extended trading cycles or adopt 
high-frequency data to further verify the propositions presented in this paper.

\section{References}

[1] Li, M. L., Chui, C. M., \& Li, C. Q. (2014). Is pairs trading profitable on china ah-share markets?.Applied Economics Letters, 21(16), 1116-1121.

[2] Rad, H., Low, R. K. Y., \& Faff, R. (2016). The profitability of pairs trading strategies: distance, cointegration and copula methods. Quantitative Finance, 16(10), 1541-1558.

[3] Ramos-Requena, J. P., Trinidad-Segovia, J. E., \& Sánchez-Granero, M. A. (2017). Introducing hurst exponent in pair trading. Physica A Statistical Mechanics \& Its Applications, 488, 39-45.

[4] Chen, C. W. S., Wang, Z., Sriboonchitta, S., \& Lee, S. (2017). Pair trading based on quantile forecasting of smooth transition garch models. North American Journal of Economics \& Finance, 39, 38-55.

[5] Elliott R J, Van Der HoekJ, Malcolm W P. (2005). Pairs trading. Quantitative Finance, 5(3), 271-276.

[6] Bertram, W. K. (2010). Analytic solutions for optimal statistical arbitrage trading. Physica A Statistical Mechanics \& Its Applications, 389(11), 2234-2243.

[7] Vidyamurthy, G. (2004). Pairs trading: quantitative methods and analysis. Pearson Schweiz Ag, 35-47.

[8] Nath, P. (2003). High frequency pairs trading with u.s. treasury securities: risks and rewards for hedge funds. Ssrn Electronic Journal.

[9] Zeng, Z., \& Lee, C. G. (2014). Pairs trading: optimal thresholds and profitability. Quantitative Finance, 14(11), 1881-1893.

[10] Laws, J. (2015). Trading and hedging the corn/ethanol crush spread using time-varying leverage and nonlinear models. European Journal of Finance, 21(4), 352-375.

[11] Göncü, A., \&Akyildirim, E. (2016). A stochastic model for commodity pairs trading. Quantitative Finance, 16(12), 1-15. 